\title{
A Case of Pulmonary Metastasis of Breast Cancer 23 Years after Surgery Accompanied with Non-Tuberculous Mycobacterium Infection
}

\author{
Yuki Yabuuchia ${ }^{\text {Takayuki Nakagawa }}{ }^{b}$ Masaki Shimanouchi ${ }^{b}$ \\ Shingo Usui ${ }^{b}$ d Kenji Hayashihara ${ }^{a}$ Shuji Oh-ishi ${ }^{a}$ Takefumi Saito ${ }^{a}$ \\ Jun Kanazawa $^{a}$ Yukiko Miura $^{a}$ Shouta Kubota ${ }^{a}$ Kai Kawashima ${ }^{a}$ \\ Takafumi Shimada $^{a}$ Hisayuki Oshima ${ }^{a}$ Hitomi Hirano ${ }^{a}$ Mizu Nonaka ${ }^{a}$ \\ Yuka Kitaoka ${ }^{a}$ Naoki Arai ${ }^{a}$ Kentaro Hyodo ${ }^{a}$ Atsuhito Nakazawa ${ }^{a}$ \\ Yuko Minamic \\ aDepartment of Respiratory Medicine, National Hospital Organization, Ibaraki Higashi \\ National Hospital, Ibaraki, Japan; ${ }^{b}$ Department of Respiratory Surgery, National Hospital \\ Organization, Ibaraki Higashi National Hospital, Ibaraki, Japan; 'Department of Pathology, \\ National Hospital Organization, Ibaraki Higashi National Hospital, Ibaraki, Japan; \\ dDepartment of Clinical Research, National Hospital Organization, Ibaraki Higashi National \\ Hospital, Ibaraki, Japan
}

\section{Keywords}

Tumour dormancy · Mycobacterium intracellulare - Caseous epithelioid cell granuloma

\begin{abstract}
Recurrence of oestrogen receptor (ER)-positive breast cancer rarely occurs postoperatively after a long period. Breast cancer cells survive and settle in distant organs in a dormant state, a phenomenon known as "tumour dormancy." Here, we present a 66 -year-old woman with recurrence of ER-positive breast cancer in the left lung 23 years after surgery accompanied with non-tuberculous mycobacterium infection (NTM). At the age of 43 years, the patient underwent a right mastectomy and adjuvant hormonotherapy to completely cure breast cancer. Twenty-three years after the operation, when the patient was 66 years old, computed tomography presented nodular shadows in the lower lobes bilaterally with bronchiectasis and illdefined satellite tree-in-bud nodules. Mycobacterium intracellulare was detected in cultured bronchoalveolar lavage fluid obtained from the left lower lobe by bronchoscopy. Rifampicin, ethambutol, and clarithromycin were started, which resulted in shrinkage of the nodule in the right lower lobe and satellite nodules; however, the nodule in the left lower lobe increased in size gradually. Wedge resection of the left lower lobe containing the nodule by video-assisted thoracoscopic surgery was performed, which demonstrated that the nodule was adeno-
\end{abstract}


carcinoma in intraoperative pathological diagnosis; therefore, a left lower lobectomy and mediastinal lymph node dissection were performed. The tumour was revealed to be consistent with recurrence of previous breast cancer according to its morphology and immunohistochemical staining. Furthermore, caseous epithelioid cell granulomas existed in the periphery of the tumour. It is reported that inflammatory cytokines induce reawakening of dormant oestrogen-dependent breast cancer and, in our case, NTM infection might have stimulated the dormant tumour cells in the lower lobe.

(C) 2020 The Author(s).

Published by S. Karger AG, Basel

\section{Introduction}

Breast cancer rarely ever relapses many years after radical mastectomy. The risk of recurrence in small, node-negative (T1N0), low-grade tumours was approximately $10 \%$ in 5-20 years [1]. There have been few reports about recurrence of breast cancer exceeding 20 years after operation [2-4]. The lung is the major site of breast cancer metastasis because it is the specific organ for dissemination of cancer cells for the development of metastasis [5]. Here, we present a case of recurrence of breast cancer in the left lower lobe 23 years after right mastectomy accompanied by non-tuberculous mycobacterium infection (NTM). Some studies have demonstrated that stromal inflammation plays an important role in the reawakening of dormant oestrogen-dependent breast cancer [6]. In our case, NTM induced chronic inflammation mediated by various cytokines, which might be associated with the awakening of dormant tumour cells.

\section{Case Presentation}

The patient was a 66-year-old woman, a never smoker. In 1993, she was diagnosed with right breast cancer at the age of 43 and a right mastectomy was performed in hospital A. The pathological diagnosis of the breast cancer was scirrhous carcinoma as pT2aN0M0, stage I from the American Joint Committee of Cancer 4th edition (AJCC), at present pT2N0M0, stage IIA from AJCC 8th edition, which was positive for oestrogen receptor (ER) and progesterone receptor (PgR), and negative for human epidermal growth factor receptor type 2 (HER2). Postoperatively, she had been medicated with doxyfluridine $800 \mathrm{mg}$ for a year and tamoxifen

Fig. 1. Chest X-ray showing a 25-mm nodule in the left middle area and a 17-mm nodule in the right lower area.

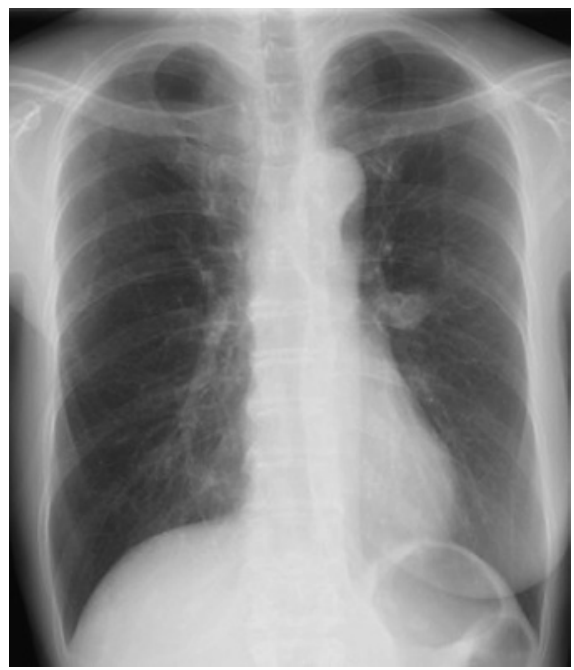




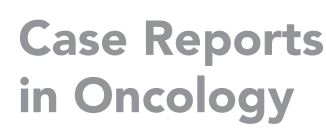

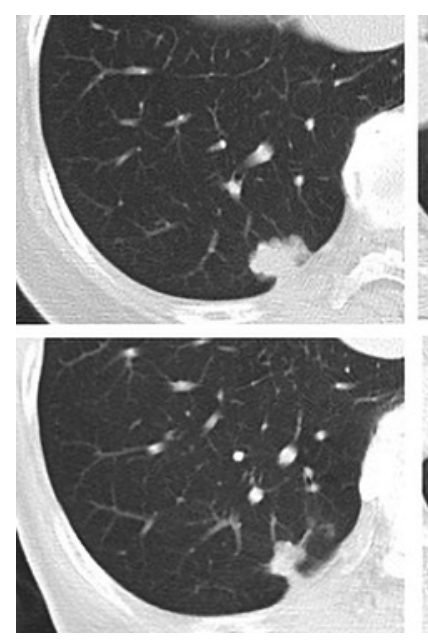
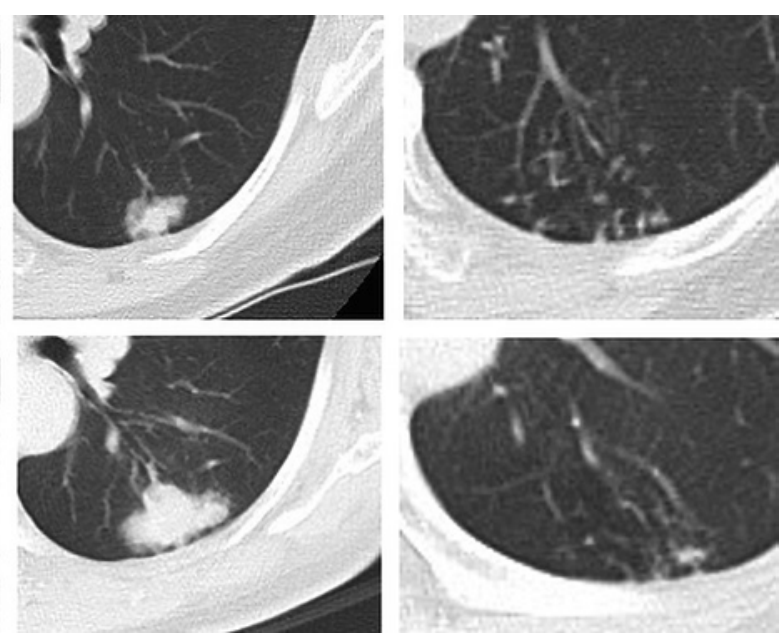

Fig. 2. Images of chest computed tomography (CT) showing a 29-mm nodule with small satellite nodules around and central bronchiectasis in the left superior segment. Furthermore, there was a 12-mm nodule in the posterior basal segment of the right lung. From a culture of bronchial lavage fluid in the superior segmental bronchus of the left lung, Mycobacterium intracellulare was detected. Medication of rifampicin, ethambutol, and clarithromycin resulted in reduction of the size of the right nodule; however, the left nodule gradually grew.

citrate $20 \mathrm{mg}$ for 6 years as adjuvant therapy. Four years of follow-up were continued after completion of adjuvant therapy, without recurrence.

In 2015, when she went for a medical check-up, a chest radiograph presented nodules in the bilateral lower lung fields. Chest computed tomography (CT) indicated solitary nodules in both lower lobes with small branching nodules and bronchiectasis around. The culture inspection of acid-fast bacterium from bronchoalveolar lavage fluid from the left lower lobe presented Mycobacterium intracellulare. She was diagnosed with NTM, and clarithromycin $600 \mathrm{mg}$, rifampicin $450 \mathrm{mg}$, and ethambutol $500 \mathrm{mg}$ were started in hospital B. In addition to streptomycin $750 \mathrm{mg}$ for 3 months, the therapy was continued for a year and a half. The size of the right nodule was reduced; however, the left nodule had grown (Fig. 1, Fig. 2). For an in-depth examination, she was admitted to our hospital. Bronchial brushing and bronchoalveolar lavage of the left nodule by bronchoscopy were performed. Although Mycobacterium intracellulare was not detected from the specimen, abrasion cytology showed class IIIB according to the Papanicolaou classification. Positron emission tomography CT (PET-CT) presented concentration of fluoro-deoxy-glucose (FDG), standard uptake value (SUV) max: 9.25 in the left nodule, but not in the right nodule and both breasts (Fig. 3). Lung cancer was suspected, and surgery was performed.

On presentation, the patient had a slight temperature $\left(37.3^{\circ} \mathrm{C}\right)$; otherwise, there were no abnormal physical findings.

All of the laboratory data were normal, and serum tumour markers and markers of infection, such as Aspergillus and Mycobacterium, were all negative.

Wedge resection of the left lower lobe by video-assisted thoracoscopic surgery was performed. The nodule was diagnosed as a malignant tumour by intraoperative pathological diagnosis. After that, a left lower lobectomy and mediastinal lymph node dissection were performed. Histologically, the lung tumour appeared medullary, follicular and papillary with necrosis, but not as lepidic growth (Fig. 4). Immunohistochemically, the tumour presented negative for thyroid transcription factor-1, ER, PgR, GATA binding protein 3, and mamma-

\section{Karger'}




\section{Case Reports in Oncology}

\begin{tabular}{l|l}
\hline Case Rep Oncol 2020;13:1357-1363 \\
\hline DOI: 10.1159/000511072 & $\begin{array}{l}\text { ○ 2020 The Author(s). Published by S. Karger AG, Basel } \\
\text { www.karger.com/cro }\end{array}$ \\
\hline
\end{tabular}

Yabuuchi et al.: Pulmonary Metastasis of Breast Cancer 23 Years after Surgery

Fig. 3. Positron emission tomography CT showing concentration of fluorodeoxy-glucose in the left nodule, but not in the right nodule.
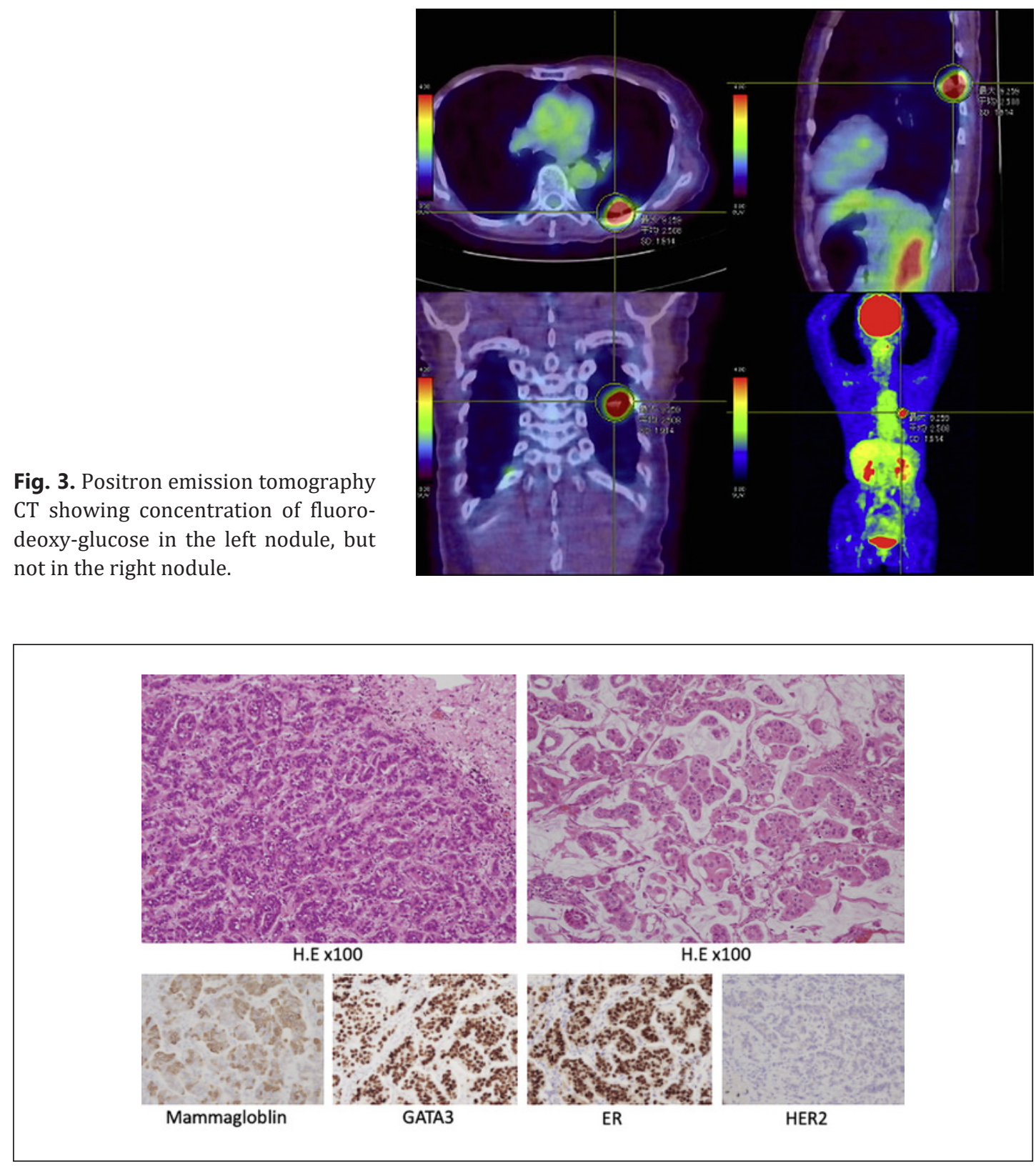

Fig. 4. Pathological features of left lower lobe, with appearing tumour growing like medullary, follicularly, and papillary, with destruction of the alveolar walls. However, the tumour did not take the form of lepidic growth. Immunohistochemistry indicated that the tumour was positive for GATA binding protein 3 (GATA3), mammagloblin, oestrogen receptor (ER), and progesterone receptor and negative for human epidermal growth factor receptor type 2 (HER2).

globin, moderately positive for p53 tumour suppressor gene, and with a HER2 score of zero (Fig. 4). On the other hand, breast cancer at 43 years had presented scirrhous carcinoma with lymphatic permeation and vascular invasion (Fig. 5). Furthermore, staining for ER and PgR were positive and HER2 was negative (Fig. 5). The histopathological presentation of the tumour was consistent with the right breast cancer at the age of 43; therefore, we diagnosed a recurrence of breast cancer in the left lower lobe. Moreover, epithelioid cell granulomas 


\section{Case Reports in Oncology}

\begin{tabular}{l|l}
\hline Case Rep Oncol 2020;13:1357-1363 \\
\hline DOI: 10.1159/000511072 & $\begin{array}{l}\text { @ 2020 The Author(s). Published by S. Karger AG, Basel } \\
\text { www.karger.com/cro }\end{array}$ \\
\hline
\end{tabular}

Yabuuchi et al.: Pulmonary Metastasis of Breast Cancer 23 Years after Surgery
Fig. 5. Pathological features of breast cancer at the age of 43 showing scirrhous carcinoma infiltrating fatty tissue, lymph ducts, and vessels around. Immunostaining of the breast cancer was positive for ER and PgR and negative for HER2. The growth morphology and patterns of immunostaining of the tumour in left lower lobe confirmed that of breast cancer at the age of 43 .

Fig. 6. Pathologically, epithelioid cell granulomas with caseous necrosis and accumulation of Langhans giant cells were detected in the tumour periphery. ZielNeelsen stains were negative; therefore, these findings suggested prior NTM infection.

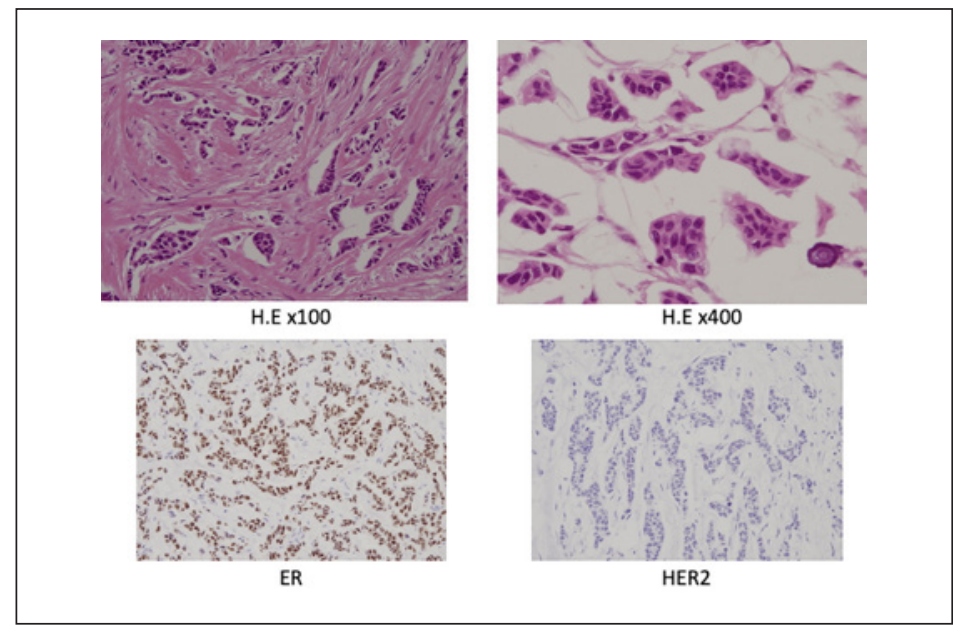

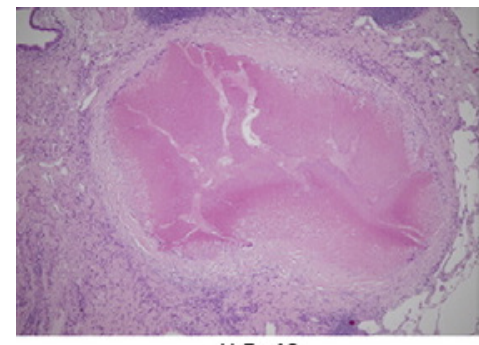

H.E $\times 40$

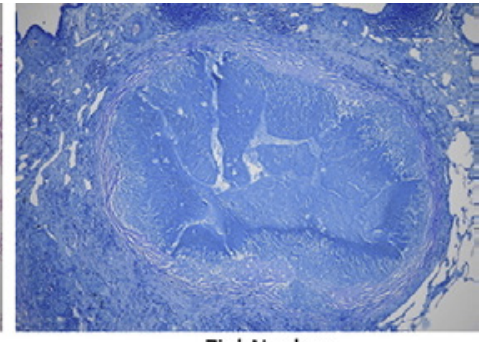

Ziel-Neelsen

with caseous necrosis and multinucleated Langhans giant cells accumulated in the tumour periphery, which suggested complication of NTM (Fig. 6). After leaving our hospital, she was readmitted to hospital A.

\section{Discussion and Conclusion}

Radiologic manifestations of NTM sometimes present solitary nodules mimicking lung carcinoma. Chest CT features of NTM show satellite lesions around the main nodule and bronchiectasis in the same segment [7]. Benign solitary lesions frequently present calcification and poor contrast enhancement; however, NTM sometimes show a lobulated border, pleural retraction and FDG uptake on PET-CT, which are common findings of malignant tumours [7]. In our case, the nodule in the left lower lobe was accompanied by satellite lesions and bronchiectasis, which suggested NTM (Fig. 2). On the other hand, the nodule also showed pleural indentation and concentration of FDG, SUV max: 9.25 (Fig. 3); moreover, brushing cytology obtained by bronchoscopy showed class IIIB according to the Papanicolaou classification, which suggested malignant disease; therefore, surgical resection was chosen. We diagnosed the left lung nodule as a recurrence of breast cancer from pathological findings of lung tissue corresponding with the morphology and patterns of immunostaining of the right breast cancer (Fig. 4, Fig. 5). Furthermore, it was suggested that NTM was complicated with breast cancer recurrence because tumours and epithelioid cell granulomas with caseous necrosis were detected in the same lobe (Fig. 6). There have been some reports of breast cancer recurrence more than 20 years later [8]; however, a case of breast cancer recurrence in the lung 
with NTM after long periods has not been presented as far as we know. In our case, NTM might have played an important role in the recurrence of breast cancer in the left lung as per the "tumour dormancy theory."

Tumour dormancy means that tumour cells remain concealed, asymptomatic, and untraceable, eventually progressing to overt cancer after a long period [9]. After resection of the tumour, the tumour cells sometimes remain in low numbers in a state of circulating tumour cells, disseminated tumour cells or micrometastases. These tumour cells are in a state of cell cycle arrest or balance between apoptosis and cell proliferation, resulting in no increase in the tumour burden [9]. The tumour cells are resistant to adjuvant chemotherapy; moreover, they evade immune surveillance [10]. Tumour dormancy is present particularly in ER-positive breast cancer, in which $20-45 \%$ of patients end up with distant recurrence of the disease years or decades after successful treatment [11]. The risk of recurrence in the first 2 years after diagnosis is higher in ER-negative than in ER-positive patients. On the other hand, more than half of metastases in ER-positive breast cancer occur 5 years or longer after diagnosis [12]. It has been reported that the expression of genes related to dormancy was significantly high in ER-positive compared to ER-negative tumours [12]. Therefore, it is important to evaluate the appropriate hormone treatment period for ER-positive patients [13]. Actually, in our case, relapse occurred in the left lung more than 20 years after mastectomy, although hormonotherapy had been performed for 6 years.

There is a correlation between chronic inflammation and recurrence of breast cancer, especially, tumour escape from dormancy induced by cytokines [14].

Tivari et al. [6] demonstrated that stromal inflammation induced the secretion of interleukin- 6 and -8, upregulated tumour necrosis factor-alpha, and activated transforming growth factor-beta, which reactivated the growth and motility of dormant ER-positive breast cancer cells. Furthermore, chronic inflammation supports angiogenesis, which is an important factor in the promotion of growth of dormant micrometastasis [14]. In our case, NTM infection in the lower lobe was accompanied by chronic inflammation with caseating granulomas, which might induce reawakening and growth of dormant cancer cells. As a limitation, we could not prove the presence of inflammatory cytokines with serum data or pathological findings. Since NTM infection is common in middle-aged women, the NTM patients with a medical history of surgery for ER-positive breast cancer need to be aware of recurrence.

\section{Acknowledgements}

We would like to acknowledge and thank Hideya Takahisa, MD, Department of Mito Saiseikai General Hospital, Ibaraki, Japan for providing the specimens and medical information.

\section{Statement of Ethics}

This study was approved by the Ibaraki Higassi National Hospital ethical committee. Written informed consent was obtained from the patient and patient's family for publication of this case report and accompanying images. A copy of the written consent is available for review by the Editor of this journal.

\section{Conflict of Interest Statement}

The authors state that they have no conflict of interest.

\section{Karger'}




\section{Case Reports in Oncology}

\begin{tabular}{l|l}
\hline Case Rep Oncol 2020;13:1357-1363 \\
\hline DOI: 10.1159/000511072 & $\begin{array}{l}\text { @ 2020 The Author(s). Published by S. Karger AG, Basel } \\
\text { www.karger.com/cro }\end{array}$ \\
\hline
\end{tabular}

Yabuuchi et al.: Pulmonary Metastasis of Breast Cancer 23 Years after Surgery

\section{Author Contributions}

Y.Y., M.N., T.N., and T.S. treated the patient. K.H., S.O., J.K., S.K., K.K., T.S., H.O., H.H., and Y.K. supervised the bronchoscopy and subsequent treatment. N.K., M.S., and S.U. performed the operation. Y.M. made a pathological diagnosis. Y.Y. drafted the initial manuscript and submitted the final manuscript. Y.M. revised the manuscript. Y.M., N.A., K.H., and A.N. critically reviewed the manuscript. All authors read and approved the final manuscript.

\section{References}

1 Pan H, Gray R, Braybrooke J, Davies C, Taylor C, McGale P, et al. 20-year risks of breast-cancer recurrence after stopping endocrine therapy at 5 years. N Engl J Med. 2017 Nov 9;377(19):1836-46.

2 Miyake M, Yamada A, Miyake K, Endo I. Esophageal metastasis of breast cancer during endocrine therapy for pleural dissemination 21 years after breast surgery: a case report. Surg Case Rep. 2019 Dec;5(1):22-7.

3 Zhijie L, Qin C, Yuanyuan Z, Xiaochuan W, Siying F, Linzhu Z. Late distant recurrence of breast carcinoma and metastasis to the main bronchus and choroid. Medicine. 2018 May;97(20):1-5.

4 Izumi C, Misawa K, Endo S, Sugiyama K, Mochizuki D, Imai A, et al. Late recurrence of breast carcinoma metastasis to the hypopharynx: a case report. SpringerPlus. 2016 May;5(599):599-7.

5 Urooj T, Wasim B, Mushtaq S, Shah S, Shah M. Cancer cell-derived secretory factors in breast cancer-associated lung metastasis: their mechanism and future prospects. Curr Cancer Drug Targets. 2019 Dec;20(3):168-86.

6 Tivari S, Lu H, Dasgupta T, De Lorenzo MS, Wieder R. Reawakening of dormant estrogen-dependent human breast cancer cells by bone marrow stroma secretory senescence. Cell Commun Signal. 2018 Aug; 16(1): 48-18.

7 Hong SJ, Kim TJ, Lee JH, Park JS. Nontuberculous mycobacterial pulmonary disease mimicking lung cancer: Clinicoradiologic features and diagnostic implications. Medicine (Baltimore). 2016 Jun;95(26):e3978-6.

8 Harada H, Yamashita Y, Tsubokawa N. Two cases of lung metastasectomies from breast cancer over 30 years after initial surgeries. Nihon kokyuki geka gakkai zasshi. 2013;27(6):104-9.

9 Gelao L, Criscitiello C, Fumagalli L, Locatelli M, Manunta S, Esposito A, et al. Tumour dormancy and clinical implications in breast cancer. Ecancermedicalscience. 2013;7(320):320-15.

10 Zhang XH, Giuliano M, Trivedi MV, Schiff R, Osborne CK. Metastasis dormancy in estrogen receptor-positive breast cancer. Clin Cancer Res. 2013 Dec 1;19(23):6389-97.

11 Masound M. Tumor dormancy and relapse: from a natural by-product of evolution to a disease state. Cancer Res. 2017 May; 77(10):2564-9.

12 Kim RS, Avivar-Valderas A, Estrada Y, Bragado P, Sosa MS, Aguirre-Ghiso JA, et al. Dormancy signatures and metastasis in estrogen receptor positive and negative breast cancer. PLoS One. 2012 Apr;7(4):e35569-8.

13 Nishimura R, Osako T, Nishiyama Y, Tashima R, Nakano M, Fujisue M, et al. Evaluation of factors related to late recurrence - later than 10 years after the initial treatment - in primary breast cancer. Oncology. 2013 Jul; 85(2):100-10.

14 Gomis RR, Gawrzak S. Tumor cell dormancy. Mol Oncol. 2017 Jan;11(1):62-78. 